it was so great and see if any further learning could be extracted for wider dissemination.

Results The FERF that was used involved a PICC line nurse who was on a bank shift, helping a doctor achieve IV access in a very tricky patient. The AI process allowed further depth to be gained in understanding the impact on staff of being valued for ones skills and being able to teach them to others as well as benefitting the patient. It also led to the idea that a difficult IV access (DIVA) pathway could be incredibly useful to guide staff on where to get help in patients who have challenging IV access. This pathway is now in development.

Conclusion The AI conversation around the FERF led to a much greater depth of understanding of the key positive issues and strengths of the event. Additionally, it provided a suggestion of an improvement pathway that will be developed that could have a positive impact on any child with DIVA. The wider impact of this positive event is far greater than that achieved purely by the FERF alone. Other departments could use this approach in the same way to further investigate FERFs.

\section{G392(P) MANAGEMENT OF ACUTE WHEEZE- WHAT DO PAEDIATRIC TRAINEES DO?}

${ }^{1} V$ Currie, ${ }^{2} \mathrm{~L}$ Duthie, ${ }^{3} \mathrm{P}$ Nagakumar. ${ }^{1}$ Paediatrics, Worcester Royal Hospital, Worcester, UK; ${ }^{2}$ Paediatric Intensive Care, Birmingham Children's Hospital, Birmingham, UK; ${ }^{3}$ Paediatric Respiratory Medicine, Birmingham Children's Hospital, Birmingham, UK

\subsection{6/archdischild-2020-rcpch.336}

Aims Current guidelines of acute management of wheeze in children are open to interpretation (Keeley:2018). Individual clinician preference and many 'local guidelines' influence the initial management by the frontline paediatric trainees. We hypothesised that there is greater variation in practice with acute preschool wheeze than the school age children with acute asthma.

Methods Online survey of paediatric trainees in a large Deanary using three clinical scenarios of children of different ages presenting with acute wheeze. Trainees were asked to select the most appropriate management plan of giving inhalers, nebulisers or 'back to back therapy'. Following reassessment trainees were then asked for the next line of treatment

Results 87 responses from ST1-ST8 trainees between March and July 2019. 85\% were managing at least one child with wheeze every day. $80 \%$ of respondents had a minimum of 3 years of paediatric experience. In a pre-school child with wheeze and saturations of $94 \%, 78 \%$ of trainees gave 10 puffs of salbutamol as initial treatment. $34 \%$ would give 2 further bronchodilators 'back to back' after initial improvement.

In both cases of older children with asthma, half of trainees gave a nebuliser an initial therapy despite the oxygen saturations $>92 \%$ at presentation. $20 \%$ of respondents understand the term 'back to back' to mean an interval of between 15 and 30 minutes.

97\% of trainees give written wheeze information to families with $87.5 \%$ opting for 3 day salbutamol weaning plan at discharge.

Conclusions Contrary to our hypothesis, the survey demonstrates that there is more consistency in the initial management of preschool wheeze compared to older children with asthma. This may reflect the service pressures to decide about admitting or discharging the child rather than an uncertainty about clinical situation.
In older children where clinical assessment is more predictable, surprisingly, half of the trainees administered nebulised bronchodilators despite normal oxygen saturations. Older children may have had inhalers for a period of time (not acknowledged in current BTS guidelines) prompting trainees to take a different approach.

Discharging children with a Salbutamol weaning plan is unique to the UK practice (Levy:2018) which needs to be addressed by prospective studies.

\section{G393(P) MANAGING THE WINTER-PRESSURE REGISTRAR ROTA: OUR EXPERIENCE}

R Kirk, P Chandershekar, G Jain. Lister Hospital, East and North Herts NHS Trust, Stevenage, UK

\subsection{6/archdischild-2020-rcpch.337}

Introduction Our DGH secured funding for a winter-pressure (WP) registrar locum shift to cover CAU for 8-hours overnight from October 2018 to March 2019. Managing the WP-rota was taken up by a paediatric registrar (overseen by a consultant) as a management and leadership QI project. We aimed to evaluate trends in how locum shifts are booked and to see if a mobile messaging application (WhatsApp) administrated by a trainee could lead to improved uptake of locum bookings.

Methods

- Utilising our network of current and previous trainees a WhatsApp 'WP group' was created to advertise and book WP-shifts (alongside medical staffing advertising via their emailing lists).

- Shifts were also open to level-1 trainees with full MRCPCH membership.

- We managed at standardised NHS-P rate.

- There were flexible start times: $10 \mathrm{pm}, 11 \mathrm{pm}$ or midnight.

- The paediatric registrar administrator was the direct clinical contact and liaised with medical staffing to organise bookings.

Results

- 96\% ( $\mathrm{N}=174)$ of WP-shifts were booked.

- 91\% $(\mathrm{N}=160)$ of WP-shifts were booked via direct communication with the registrar administrator through WhatsApp.

- $9 \%(\mathrm{~N}=14)$ of WP-shifts were booked as a result of medical staffing emailing advertisements alone.

- 57\% (N=99) of WP-shifts were booked with more than 4 weeks' notice.

- 19 doctors booked shifts. 100\% of doctors booked more than one shift.

- Informal feedback from registrars was collected; both local trainees and locums felt the WP-rota made the department feel safer and had improved staff morale.

Take Home Messages

- Anecdotally locums liked the flexibility and convenience of choosing their start time.

- Good communication with a personal touch is key. Using a mobile chat application is an efficient way to advertise shifts, answer queries and confirm bookings.

- The locums valued the ease of communication with a clinical contact who would liaise and book shifts with medical staffing on their behalf. Locum doctors and local trainees appreciated a clinical person who could relate to them overseeing the WP-rota. 